\title{
Evaluating the use of enforced clozapine in an Australian forensic psychiatric setting: two cases John Kasinathan* and Tony Mastroianni
}

\author{
Address: Long Bay Hospital, PO Box 150, Matraville 2036, Australia \\ * Corresponding author
}

\author{
from WPA Thematic Conference. Coercive Treatment in Psychiatry: A Comprehensive Review \\ Dresden, Germany. 6-8 June 2007 \\ Published: 19 December 2007 \\ BMC Psychiatry 2007, 7(Suppl I):PI3 doi:I0.I 186/I47I-244X-7-SI-PI3
}

This abstract is available from: http://www.biomedcentral.com/I47I-244X/7/SI/PI3

(c) 2007 Kasinathan and Mastroianni; licensee BioMed Central Ltd.

\section{Background}

To evaluate the administration, outcomes and ethical issues of enforced clozapine in two patients with treatment-resistant schizophrenia/schizoaffective disorder in a forensic psychiatric setting, who were refusing oral clozapine.

\section{Methods}

"Enforced clozapine" implied that if a patient refused oral clozapine they received intramuscular clozapine at half the expected oral dose. The case series involved 12 months follow-up. Baseline and monthly measures included the Brief Psychiatric Rating Scale (BPRS), Clinical Global Impressions (CGI), Global Assessment of Functioning (GAF), Insight and Treatment Attitudes Questionnaire (ITAQ) and Quality of Life Self-Assessment Inventory (QLS-100). Routine haematological, metabolic and cardiac monitoring was informed by established clinical guidelines. Statistical analysis utilized t-Tests and Pearson's correlation coefficient.

\section{Results}

Enforced clozapine was reasonably well tolerated with rapid acceptance of oral clozapine. By 12 months, statistically significant improvements were seen in BPRS ( $\mathrm{p}=$ $0.05)$, CGI $(\mathrm{p}=0.04)$ and GAF $(\mathrm{p}=0.03)$. Improvements in ITAQ and QLS-100 were not statistically significant. BPRS and CGI were strongly negatively correlated with the length of time on enforced clozapine, clozapine dose and clozapine serum levels. GAF, QLS-100 and to a lesser extent ITAQ were positively correlated with those factors.

\section{Conclusion}

Enforced clozapine is a viable short-term treatment option in treatment-resistant patients who are refusing oral clozapine. It resulted in significantly reduced psychotic symptoms, reduced dangerousness, a better quality of life, improved insight into their illness and long-term acceptance of oral clozapine. Unfortunately recent global non-production of parenteral clozapine means that this promising intervention in selected treatment-resistant patients cannot be further evaluated and judiciously implemented. 